\title{
Systemic capillary leak syndrome after surgery for colon cancer perforating peritonitis treated using plasma filtration with dialysis
}

\author{
Ginga Suzuki ${ }^{1}$, Ryo Ichibayashi ${ }^{1}$, Saki Yamamoto ${ }^{1}$, Hibiki Serizawa ${ }^{1}$, Yoshimi Nakamichi ${ }^{1}$, \\ Masayuki Watanabe ${ }^{1}$, and Mitsuru Honda ${ }^{1}$ \\ ${ }^{1}$ Toho University Omori Medical Center
}

November 5, 2020

\author{
Abstract \\ We present a case of Systemic capillary leak syndrome in a patient who underwent surgery for colon cancer perforating peritonitis. \\ We treated the patient with plasma filtration with dialysis. \\ Manuscript category: Case report \\ Title: Systemic capillary leak syndrome after surgery for colon cancer perforating peritonitis treated using \\ plasma filtration with dialysis \\ Running title: SCLS treated with PDF \\ Authors: Ginga Suzuki, Ryo Ichibayashi, Saki Yamamoto, Hibiki Serizawa, Yoshimi Nakamichi, Masayuki \\ Watanabe, Mitsuru Honda \\ Authors \\ Ginga Suzuki \\ Institute: Emergency and Critical Care Center, Toho University Omori Medical Center \\ Country: Japan \\ Email: ginga.suzuki@med.toho-u.ac.jp \\ Ryo Ichibayashi \\ Institute: Emergency and Critical Care Center, Toho University Omori Medical Center \\ Country: Japan \\ Email: ryou.ichibayashi@med.toho-u.ac.jp \\ Saki Yamamoto \\ Institute: Emergency and Critical Care Center, Toho University Omori Medical Center \\ Country: Japan \\ Email: saki.yamamoto@med.toho-u.ac.jp \\ Hibiki Serizawa \\ Institute: Emergency and Critical Care Center, Toho University Omori Medical Center
}


Country: Japan

Email: serihibi@gmail.com

Yoshimi Nakamichi

Institute: Emergency and Critical Care Center, Toho University Omori Medical Center

Country: Japan

Email: yoshimi.nakamichi@med.toho-u.ac.jp

Masayuki Watanabe

Institute: Emergency and Critical Care Center, Toho University Omori Medical Center

Country: Japan

Email: masayuki.watanabe@med.toho-u.ac.jp

Mitsuru Honda

Institute: Emergency and Critical Care Center, Toho University Omori Medical Center

Country: Japan

Email: mhonda@med.toho-u.ac.jp

Institution (of all authors): Critical Care Center, Toho University Omori Medical Center

Address: 6-11-1 Omori-Nishi, Otaku, Tokyo, Japan

Tel: 03-3762-4151, Fax: 03-3762-4129, E-mail:ginga.suzuki@med.toho-u.ac.jp

Corresponding author: Ginga Suzuki

Critical Care Center, Toho University Omori Medical Center

6-11-1 Omori-Nishi, Otaku, Tokyo, Japan

Tel: 03-3762-4151, Fax: 03-3762-4129, E-mail:ginga.suzuki@med.toho-u.ac.jp

\section{Abstract}

We present a case of Systemic capillary leak syndrome in a patient who underwent surgery for colon cancer perforating peritonitis. We treated the patient with plasma filtration with dialysis.

Keywords: systemic capillary leak syndrome, SCLS, plasma filtration with dialysis, PDF

\section{Key Clinical Message}

The use of plasma filtration with dialysis (PDF) may be considered when treating the acute phase of Systemic capillary leak syndrome (SCLS). To the best of our knowledge, this is the first report using PDF for SCLS.

\section{Background}

Systemic capillary leak syndrome (SCLS) is a very serious condition of unknown cause, first reported by Clarkson in 1960 (1). The characteristics of SCLS are hypotension, hemoconcentration, and low plasma albumin levels; however, there are no clear diagnostic criteria (2-13). Certain autoimmune systems may be involved in the disease. Various treatments have been attempted, including fluid resuscitation, renal replacement therapy, corticosteroid administration, high-dose intravenous immunoglobulin (IVIG), and plasma exchange (PE) (4-6). We present a case of SCLS that developed after surgery for colon cancer perforating peritonitis wherein we treated the patient with plasma filtration with dialysis (PDF). Moreover, we obtained new information about this condition during the course of treatment. 


\section{Case presentation}

An 84-year-old woman was admitted to the general ward of our hospital with the chief complaints of stomachache and diarrhea. She was a known case of myasthenia gravis; she had undergone thymoma extraction 6 years ago, after which she had started taking prednisolone $6 \mathrm{mg}$ /day and tacrolimus $3 \mathrm{mg} /$ day. On admission, computed tomography (CT) showed ascending colon cancer. Three days later, she underwent colon stenting with an endoscope. She complained of feverishness and a severe stomachache that night. The CT scan was re-examined and free air was found in the abdominal cavity. She was immediately taken in for emergency surgery. She underwent a right hemicolectomy and ileostomy through open surgery.

After surgery, she was admitted to the intensive care unit (ICU), where she was orally intubated and ventilated. Extracellular fluid was administered at $200 \mathrm{~mL} / \mathrm{h}$ and noradrenaline was administered at $0.3 \gamma$. Her vital signs were: body temperature $35.8^{\circ} \mathrm{C}$, blood pressure $108 / 65 \mathrm{mmHg}$, pulse $122 / \mathrm{min}$, respiratory rate $15 /$ min under sedation. Meropenem $3 \mathrm{~g} /$ day and daptomycin $350 \mathrm{mg} /$ day were used as antibiotics. Table 1 shows the blood test parameters on admission to the ICU. The blood pressure started dropping 5 hours after the operation; the fluid volume was increased to $500 \mathrm{~mL} / \mathrm{h}$, but the blood pressure did not rise. Therefore, 7 hours after the operation, continuous administration of hydrocortisone $(200 \mathrm{mg} /$ day $)$ and continuous hemodiafiltration (CHDF) were introduced. We were using the EV1000? (Edwards Life Science, Ltd) for hemodynamic monitoring. Based on the data provided by the EV1000?, especially the global end-diastolic volume index (GEDI), up to 17 hours after the operation, we gradually reduced the infusion volume. The day after the operation, the infusion was $200 \mathrm{~mL} / \mathrm{h}$. By the 2 nd postoperative day, the infusion could be reduced to $100 \mathrm{~mL} / \mathrm{h}$; however, after a while, the blood pressure began to decrease. The infusion volume was therefore again increased to $500 \mathrm{~mL} / \mathrm{h}$. On the $3 \mathrm{rd}$ postoperative day, it was not possible to reduce the amount of the fluid, and we had to increase the infusion to $1000 \mathrm{~mL} / \mathrm{h}$. Although her condition was initially attributed to uncontrolled sepsis, her C-reactive protein level, white blood cell count, and procalcitonin level had improved on the third postoperative day; moreover, no bacteria were detected in the postoperative culture specimens. In addition, the serum albumin level was extremely low $(0.7 \mathrm{mg} / \mathrm{dL})$, due to which we suspected SCLS for the first time. We conducted blood tests, which revealed increased level of kappa light chain M protein $(9.6 \mathrm{mg} / \mathrm{dL})$. Urinary Bence Jones protein was negative and C-1 esterase inhibitor level was low (Table 1). Based on these results, we diagnosed SCLS. At the time of diagnosis, her condition was quite critical, with the blood pressure dropping to $48 / 30 \mathrm{mmHg}$ despite an infusion speed of $1000 \mathrm{~mL} / \mathrm{h}$. Therefore, PDF was introduced in preparation for administration of a high dose of IVIG $(0.4 \mathrm{~g} / \mathrm{kg} / \mathrm{day})$.

After PDF was started, her blood pressure gradually increased. About 2 hours later, her blood pressure was $132 / 70 \mathrm{mmHg}$. Soon after that, IVIG was started. The infusion speed was gradually decreased, and 9 hours after PDF started, the infusion speed was $60 \mathrm{~mL} / \mathrm{h}$. The PDF was continued for 39 hours and we continued CHDF. The patient's condition was stable for approximately 37 hours after PDF was stopped. Subsequently, her blood pressure gradually decreased. We reintroduced PDF as soon as we expected the SCLS to relapse. In addition, steroid pulse therapy (methylprednisolone $1 \mathrm{~g} /$ day) was also initiated, as PDF alone raised concerns about the patient relapsing. Her condition began to improve and stabilized again.

However, after the PDF was stopped, the patient reverted to her initial state in approximately 2 days. We therefore used PDF for the third time. PDF was effective, but the patient relapsed a few days later. PDF was effective, but the patient relapsed a few days later and died. The ICU course is shown in Fig. 1.

\section{Discussion and Conclusions}

We obtained two new findings from this case. First, to the best of our knowledge, this is the first case to use PDF for SCLS. Second, we determined that the substances that are therapeutic targets for SCLS are likely to be included in the medium molecule region.

We introduced PDF to treat SCLS. PDF is a blood purification method in which a dialysate is recirculated to the outside of a hollow fiber while performing plasma exchange with fresh frozen plasma (FFP) or an albumin solution using a membrane type plasma separator Evacuer? Plus (Kawasumi Chemical Industry, Ltd.) EC2A filter. PDF is said to be able to remove small and medium-sized harmful substances while retaining 
useful substances in the large molecule region, such as immunoglobulin and coagulation factors. Therefore, the major advantage of PDF compared to PE is that it can reduce the use of FFP $(14,15)$. Reducing the use of FFP can lower the cost of treatment and reduce the risk of blood transfusion complications.

In this case, PDF showed a remarkable effect, due to which we can surmise that substances within the treatment target of PDF may be the cause of SCLS. In Japan, PDF is treated as PE for insurance claims, so the use of PDFs is limited to those with PE insurance coverage. However, this patient also had myasthenia gravis and was covered by insurance for PE, due to which performing PDF was not a problem. In other circumstances, the cost can be an issue with using PDF. Therefore, unless the physician is very confident about the diagnosis, PDF should be used with extreme caution.

The findings obtained in this case can be useful to plan treatment for SCLS in future cases. If substances in the middle molecular area are responsible for increased vascular permeability, immunoglobulin and $\mathrm{M}$ protein will not be directly responsible. There are reports of vascular endothelial growth factor and angiopoietin 2 as substances in the middle molecular region and they reflected the clinical course(13). Together with the results of this study, it may be that such substances are the cause of SCLS.

SCLS is a rare syndrome, difficult to diagnose, and has an uncertain etiology. Although our patient did not show any hemoconcentration, she was diagnosed as having SCLS due to prominent hypotension and decrease in GEDI and hypoalbuminemia. In this case, the recurrence of sepsis could not be ruled out, but there were no major problems during surgery, the appropriate antibiotics had been administered, and blood test results had showed improvement. Finally, we could confidently diagnose SCLS based on the presence of monoclonal M protein. Other differential diseases, such as ovarian hyper-stimulation syndrome and hereditary angioedema were ruled out based on serum estradiol level and $\mathrm{C} 1$ esterase inhibitor level, respectively. Although there have been reports of SCLS complicated with cancer (8), the relationship between cancer and SCLS is unclear. In addition, SCLS occurring with myasthenia gravis has not been reported in the past, and it is unclear whether the two diseases are related. In this case, it was not possible to completely improve the vascular permeability. The patient may not have been completely cured due to persisting malignancy or metastasis. However, PDF may be useful in overcoming the acute phase of SCLS.

In summary, an 84-year-old woman with a history of myasthenia gravis underwent surgery for colon cancerrelated perforating peritonitis. After surgery, SCLS developed and was treated using PDF. PDF was considered to be an effective treatment that covers the therapeutic target of SCLS. The use of PDF may be considered when treating the acute phase of SCLS. To the best of our knowledge, this is the first report using PDF for SCLS.

\section{List of abbreviations}

SCLS, systemic capillary leak syndrome

PDF, plasma filtration with dialysis

IVIG, intravenous immunoglobulin

PE, plasma exchange

$\mathrm{CT}$, computed tomography

ICU, intensive care unit

CHDF, continuous hemodiafiltration

GEDI, global end-diastolic volume index

FFP, fresh frozen plasma

\section{Conflict of interest}

Not applicable. 


\section{Ethics approval}

We got the patient's family consent for this report.

\section{Funding information}

Not applicable.

\section{Acknowledgments}

The authors are greatly indebted to all the personnel at the Critical Care Center, Toho University Omori Medical Center.

\section{Authors contributions}

G.S. wrote and drafted the manuscript. G.S., R.I., S.Y., H.S., Y.N., M.W., and M.H. helped to draft the manuscript. All authors read and approved the final manuscript

\section{References}

1. Clarkson B, Thompson D, Horwith M, et al: Cyclical edema and shock due to increased capillary permeability. Am J Med. 1960; 29: 193-216.

2. Pineton de Chambrun M, Gousseff M, Mauhin W, et al: Intravenous Immunoglobulin Improve Survival in Monoclonal Gammopathy-Associated Systemic Capillary-Leak Syndrome. Am J Med. 2017; 130: 1219 e19- e27.

3. Asmundsson ASE, Bjorklund AR, Fisher GA, et al: Diagnosis of Systemic Capillary Leak Syndrome in a Young Child Treated with Intravenous Immunoglobulin in the Acute Phase. J Pediatr Care. 2018; 7: $94-6$

4. Hikasa Y, Hayashi M, Suzuki S, et al: A Case of Refractory Systemic Capillary Leak Syndrome (Clarkson's Disease) during Pregnancy. Acta Med Okayama. 2016; 70: 497-501.

5. Iwasa T, Ohashi H, Kihara K, et al: 10-year-old girl with life-threatening idiopathic systemic capillary leak syndrome: a case report. BMC Pediatr. 2014; 14: 137.

6. Kapoor P, Greipp PT, Schaefer EW, et al: Idiopathic systemic capillary leak syndrome (Clarkson's disease): the Mayo clinic experience. Mayo Clin Proc. 2010; 85: 905-12.

7. Ebdrup L, Druey KM, Druey K, et al: Severe capillary leak syndrome with cardiac arrest triggered by influenza virus infection. BMJ Case Rep. 2018.

8. Lourdes LS, Al-Quran SZ, Dang NH, et al: Systemic Capillary Leak Syndrome as an Initial Presentation of ALK-Negative Anaplastic Large Cell Lymphoma. Case Rep Hematol. 2012; 2012: 954201.

9. Tokura M, Niwano T, Nagasaka K, et al: Successful Treatment of S jogren's Syndrome Presenting as a Condition Similar to Chronic Capillary Leak Syndrome Using Combination Therapy with High-Dose Intravenous Immunoglobulin and Glucocorticoid. Case Rep Rheumatol. 2019; 2019: 4865024.

10. Saugel B, Umgelter A, Martin F, et al: Systemic Capillary Leak Syndrome associated with hypovolemic shock and compartment syndrome. Use of transpulmonary thermodilution technique for volume management. Scand J Trauma Resusc Emerg Med. 2010; 18: 38.

11. Druey KM, Parikh SM: Idiopathic systemic capillary leak syndrome (Clarkson disease). J Allergy Clin Immunol. 2017; 140: 663-70.

12. Pratesi A, Valoti P, Baldasseroni S, et al: Sudden cardiac arrest in a 73-year-old woman caused by systemic capillary leak syndrome. Intern Emerg Med. 2016; 11: 719-20.

13. Xie Z, Ghosh CC, Patel R, et al: Vascular endothelial hyperpermeability induces the clinical symptoms of Clarkson disease (the systemic capillary leak syndrome). Blood. 2012; 119: 4321-32.

14. Eguchi Y: Plasma dia-filtration for severe sepsis. Contrib Nephrol. 2010; 166: 142-9.

15. Mori T, Eguchi Y, Shimizu T, et al: A case of acute hepatic insufficiency treated with novel plasmapheresis plasma diafiltration for bridge use until liver transplantation. Ther Apher. 2002; 6: 463-6.

\section{Figure Legend}


Figure 1: Clinical course in the ICU. Abbreviations; pulse: steroid pulse (methylprednisolone $1 \mathrm{~g} /$ day), MEPM: meropenem, DAP: daptomycin, IVIG: intravenous immunoglobulin, PDF: plasma filtration with dialysis

Figure 1. Clinical course in the ICU

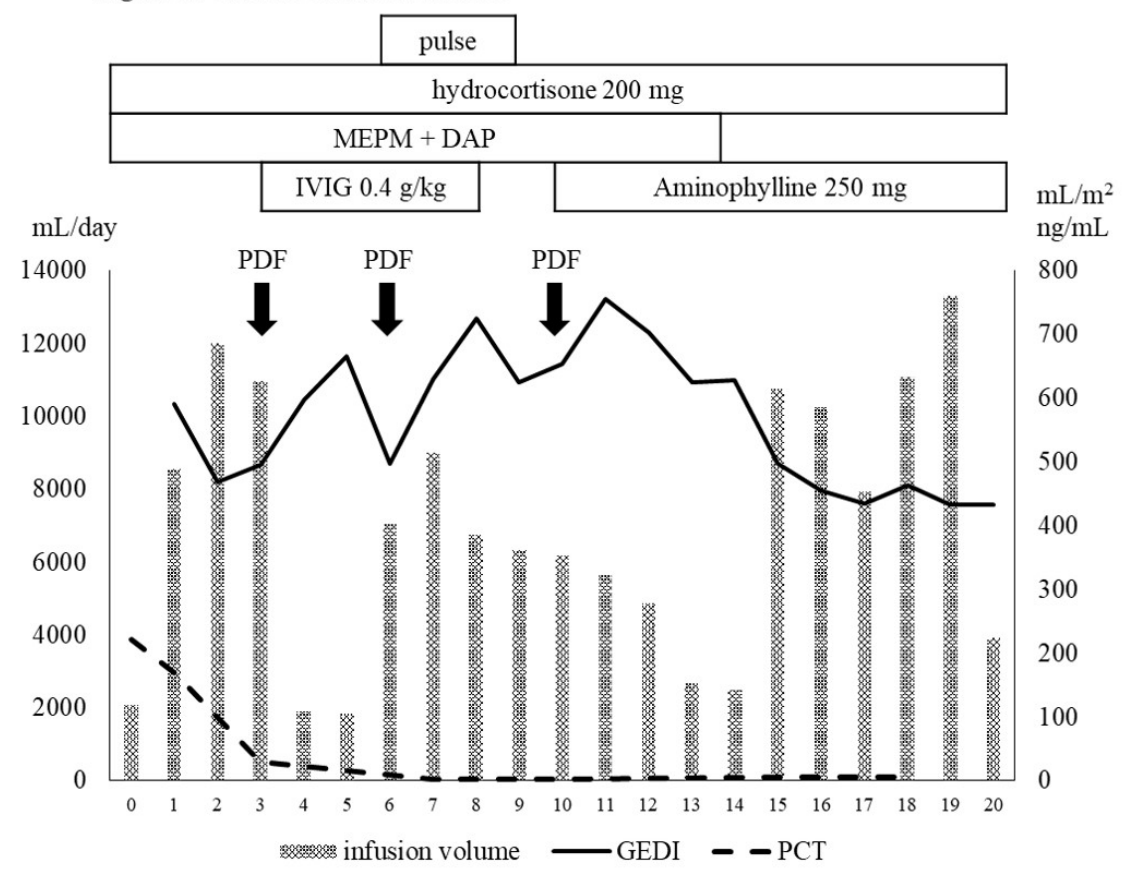

\section{Hosted file}

table1.pdf available at https://authorea.com/users/373224/articles/490988-systemic-capillaryleak-syndrome-after-surgery-for-colon-cancer-perforating-peritonitis-treated-usingplasma-filtration-with-dialysis 